\title{
Unusual case of a myxoma atrial left presenting with recurrent monoparesis
}

\author{
Gabriel E. Pérez Baztarrica, Andres Wong Baros, Nadia \\ Chiriboga, Andres Felipe Gil, Jenny Ramos Negrete, \\ Xavier Centeno Cevallos, Marco José Maldonado de la \\ Torre, Michaelly Erique, Ines Hernàndez Aviles, Reneiro \\ Velez Solorzano, Ariot Vinicio Sancan Moran, Fabio \\ Oscar Domborovski Goncalves and Rafael Porcile
}

Department of Cardiology and Physiology, Universitary Hospital, Universidad Abierta Interamericana, Faculty of Medicine,

Buenos Aires, Argentina

\section{Address for correspondence:}

Gabriel Pérez Baztarrica

Portela 2975 (|437)

Buenos Aires

Argentina

Email:

gpbaztarrica@yahoo.com

\section{INTRODUCTION}

Pure monoparesis is a rare condition characterised by weakness limited to one limb without any other neurological deficits. Isolated monoparesis is a rare symptom in patients with ischaemic stroke.(I) The aetiologies of stroke causing pure brachial monoparesis are varied and include carotid artery disease, cardiac embolism, and lobar cerebral haemorrhage. ${ }^{(2)}$ Usually, embolism is described from cardiac or large artery disease. Atrial fibrillation (AF) is the most common cause of cardiogenic emboli in stroke. Myxoma is responsible only in a few cases. We describe a case of a patient who presented with recurrent pure left brachial monoparesis as a manifestation of cardiac myxoma.

\section{CASE PRESENTATION}

A 52-year-old man with a history of arterial hypertension presented with 2 episodes of left brachial monoparesis that had begun 2 days before, and the last episode 6 hours before admission. On admission, he had systolic blood pressure (BP) of $155 \mathrm{mmHg}$ and diastolic BP of $95 \mathrm{mmHg}$, and a heart rate of 90/min. On exam, he had left brachial monoparesis with distal predominance. Muscle tone was found to be decreased and he presented with osteotendinous hyporeflexia in the left upper limb. Sensation, power, trophism and cranial nerves were without alteration. Laboratory results showed leukocytosis and

\section{ABSTRACT}

Usually, embolism is described from cardiac or large artery disease. Atrial fibrillation is the most common cause of cardiogenic emboli in stroke. Myxoma is responsible in only a few cases. We describe here a case of a patient who presented with recurrent pure left brachial monoparesis as a manifestation of a cardiac myxoma. SAHeart 2019;16:336-337

increased erythrocyte sedimentation. A brain computed tomography (CT) scan without intravenous contrast revealed an infarct in the territory of the cortical branch of the middle cerebral artery on the right side. The patient showed clinical improvement, with a complete recovery at the end of the fifth day. Transthoracic echocardiography revealed a mobile solid mass in the left atrium measuring $5.9 \mathrm{~cm} \times 4.1 \mathrm{~cm}$, prolapsing through the mitral valve in diastole (Figure |A). Thoracic CT showed a large filling defect in the left atrium (Figure IB). He was also referred for surgical resection of the atrial myxoma and a pathological diagnosis of myxoma was established (Figures IC-D). The patient did not have any postoperative complications and recovered without any neurological deficits. The patient was discharged from hospital well, with a planned follow-up visit to cardiology.

\section{DISCUSSION}

$A F$, valvular heart disease, infective endocarditis and cardiac myxoma are major sources of cerebral emboli. Myxoma is a benign tumor most commonly in the heart. The classic symptom triad includes obstructive, embolic and constitutional manifestations. The most common neurological manifestation is cerebral infarction. Up to $30 \%$ of patients with atrial myxomas suffer from embolic stroke, ${ }^{(2-3)}$ more often in younger adults. ${ }^{(4)}$ Treatment of acute ischaemic strokes caused by embolic atrial myxoma is controversial, largely because the embolus could be composed of the tumor itself or thrombus, adhered thrombotic material, or a combination of both. ${ }^{(3)}$ However, in patients who present with relevant neurological deficits within the time-window for thrombolytic treatments, thrombolysis may be an alternative to the mechanical thrombectomy - also because the diagnosis of cardiac embolism is rarely made before the treatment. ${ }^{(5)}$ Cardiac myxoma has a high tendency to pro- 
duce disabling neurological complications due to the risk of embolic episodes, which emphasise the need for its prompt surgical excision - as soon as the diagnosis is confirmed. ${ }^{(6)}$

\section{CONCLUSION}

Cardiac myxoma stroke is rare, but must be excluded in younger adults with a stroke or transient ischaemic attack. For improved diagnostic accuracy, brain CT and/or MRI and echocardiography are recommended for young stroke patients. Thrombolytic therapy may be an alternative. An early surgical resection of cardiac myxoma is recommended in patients without a large territory cerebral infarct to prevent potential consequences.

Conflict of interest: none declared.
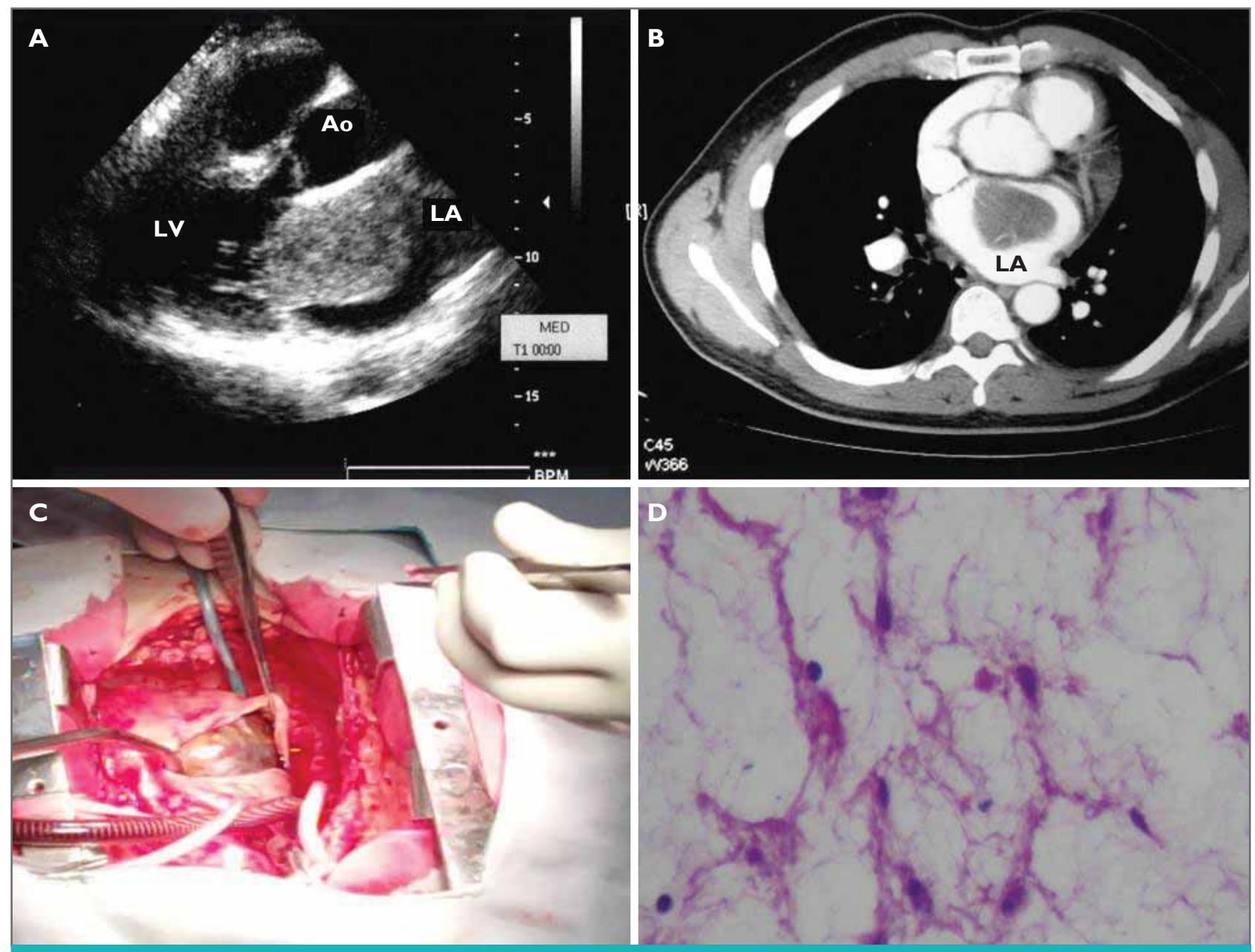

FIGURE IA: Transthoracic echocardiogram in parasternal long axis view showing mobile solid mass in the left atrium measuring $5.9 \mathrm{~cm} \times 4$. l $\mathrm{cm}$. IB: Contrast-enhanced CT angiogram image of the chest shows a large filling defect in the left atrium.

IC: Intraoperative picture showing solid tumor occupying the entire left atrium.

ID: Histopathological study demonstrated cell with a starry appearance, with eosinophilic cytoplasm and an ovoid nucleus in myxoid strom.

Ao $=$ aortic, $L V=$ left ventricular, $L A=$ left atrial. 